\title{
HOMOMORPHISMS AND RELATED CONTRACTIONS OF GRAPHS
}

\section{ROBERT D. GIRSE and RICHARD A. GILLMAN}

\author{
Department of Mathematics \\ Idaho State University \\ Pocate110, ID 83209
}

(Received August 6, 1985 and in revised form July 16, 1986)

ABSTRACT. For every homomorphism $\phi$ of a graph $G$ there exists a contraction $\theta_{\phi}$ on $\bar{G}$, the complement of $G$. Here we study the graph equation $\phi(G)=\theta_{\phi}(\bar{G})$. In the course of our work we show that Hadwiger's Conjecture is true for every self-complementary graph.

. KEYS WORDS AND PHRASES. Homomorphisms of graphs, contractions of graphs, selfcomplementary graph.

1980 AMS SUBJECT CLASSIFICATION CODE. $05 \mathrm{C} 15$.

\section{INTRODUCTION.}

By a graph of order $n$ we mean a set $V(G)$ of $n$ vertices together with a set $E(G)$ of unordered pairs of distinct vertices in $V(G)$ called edges. A graph $G$ is isomorphic to a graph $\mathrm{H}$ if there is a bijection from $\mathrm{V}(\mathrm{G})$ onto $\mathrm{V}(\mathrm{H})$ which preserves both adjacency and non-adjacency, in which case we will write $G=\mathrm{H}$. A graph with the property that $G=\bar{G}$, where $\bar{G}$ denotes the complement of $G$, is called self-complementary.

An elementary homomorphism of a graph $G$ is the identification of two non-adjacent vertices of $G$, and a homomorphism is a sequence of elementary homomorphisms. Thus a homomorphism of a graph $G$ onto a graph $H$ is a function $\phi$ from $V(G)$ onto $V(H)$ such that whenever $u$ and $v$ are adjacent in $G, \phi(u)$ and $\phi(v)$ are adjacent in $H$. Likewise, an elementary contraction of a graph $G$ is the identification of two adjacent vertices of $G$, and a contraction is a sequence of elementary contractions. Thus for every homomorphism $\phi$ of $G$ there is a contraction $\theta_{\phi}$ of $\bar{G}$ that we may construct as follows: $\phi$ is a sequence of elementary homomorphisms $\varepsilon_{1}, \varepsilon_{2}, \ldots, \varepsilon_{n}$ each of which identifies two non-adjacent vertices in $G$, so we let $\theta_{\phi}$ be the sequence of elementary contractions $\theta_{1}, \theta_{2}, \ldots, \theta_{n}$ such that $\theta_{i}$ identifies the same vertices in $\bar{G}$ that $\varepsilon_{i}$ identifies in $\mathrm{G}$.

Recently [1] the graph equation $\overline{\phi(G)}=\theta_{\phi}(\bar{G})$ was studied. Here we consider a similar equation, namely,

$$
\phi(G)=\theta_{\phi}(\bar{G})
$$

We will employ the following notation as the need arises: $\rho_{G}(u)$ will denote the valency of the vertex $u$ in $G$ and $A_{G}(u)$ will be the set of all vertices in $G$ that are 
adjacent to $u$. Thus $\rho_{G}(u)=\left|A_{G}(u)\right|$, where $\left|A_{G}(u)\right|$ is the cardinality of the set $A_{G}(u)$. As usual $X(G)$ will denote the chromatic number of $G$.

2. SOME GENERAL RESULTS.

THEOREM 1. There is no graph $G$ of order $n>1$ such that (1.1) is satisfied for every homomorphism $\phi$ of $G$.

If $G$ is not self-complementary then the identity homomophism and its related null contraction suffice to satisfy the theorem. We will postpone the remainder of the proof until section 3, where we restrict our attention to results on selfcomplementary graphs.

THEOREM 2. If there exists a homomorphism $\phi$ of $G$ that satisfies (1.1) then:

(a) $\bar{G}$ must be connected.

(b) $\bar{G}$ cannot be a tree of order $n \geqq 5$.

PROOF. (a) If $\bar{G}$ is not connected, then no contraction of $\bar{G}$ is connected. However, $\bar{G}$ not connected implies $G$ is connected, thus every homomorphic image of $G$ will be connected.

(b) If $\bar{G}$ is a tree of order $\mathrm{n} \geqq 5$ then $G$ and every homomophic image of $G$ contains $K_{3}$, the complete graph on three vertices, as a subgraph. But every contraction of $\bar{G}$ will be a tree and so cannot contain $K_{3}$ as a subgraph.

THEOREM 3. If $\chi(G)=2$ and $\bar{G}$ is connected then there exists a homomorphism $\phi$ such that $\phi(G)=K_{2}=\theta_{\phi}(\bar{G})$.

PROOF. Since $\bar{G}$ is connected, the image of $\bar{G}$ under any contraction will be connected. Thus every contraction of $\bar{G}$ onto two points must have $K_{2}$ as its image. From [3] we know that there exists a homomorphism $\phi$ of $G$ such that $\phi(G)=K_{X(G)}$. Hence, using this homomorphism and its related contraction, we have $\phi(G)=K_{2}=\theta_{\phi}(\bar{G})$.

Since every homomorphism is a sequence of elementary homomorphisms, we now turn our attention to the equation

$$
\varepsilon(G)=\theta_{\varepsilon}(\bar{G}) \text {, }
$$

where $\varepsilon$ is an elementary homomorphism.

THEOREM 4. If G satisfies (2.1) then

$$
x(\bar{G})-2 \leqq x(G) \leqq x(\bar{G})+1 .
$$

PROOF. In [3] Harary et. al. proved the following inequalities:

$$
\begin{aligned}
& \chi(G) \leqq \chi(\varepsilon(G)) \leqq \chi(G)+1 \\
& \chi(\bar{G})-1 \leqq \chi\left(\theta_{\varepsilon}(\bar{G})\right) \leqq \chi(\bar{G})+1 .
\end{aligned}
$$

Since (2.1) holds we have $\chi(\varepsilon(G))=\chi\left(\theta_{\varepsilon}(\bar{G})\right)$, and so from the first inequality $x\left(\theta_{\varepsilon}(\bar{G})\right)$ equals either $\chi(G)$ or $\chi(G)+1$. Putting these values into the second inequality above yields:

$$
\begin{aligned}
& \chi(\bar{G})-1 \leqq \chi(G) \leqq \chi(\bar{G})+1 \\
& \chi(\bar{G})-2 \leqq \chi(G) \leqq \chi(\bar{G}),
\end{aligned}
$$


which completes the proof.

The following result will be needed in the next section.

LEMMA 1. Let $\mathrm{G}$ be of order $\mathrm{n}$ and $\varepsilon$ an elementary homomorphism that identifies $u_{1}$ and $u_{2}$ in $G$. If $(2.1)$ holds then

$$
\left|A_{G}\left(u_{1}\right) \cap A_{G}\left(u_{2}\right)\right|=\left|A_{\bar{G}}\left(u_{1}\right) \cap A_{\bar{G}}\left(u_{2}\right)\right|+1+2|E(G)|-\frac{n(n-1)}{2} .
$$

PROOF. If $\varepsilon$ identifies $u_{1}$ and $u_{2}$ in $G$ we have $|E(\varepsilon(G))|=|E(G)|-\left|A_{\bar{G}}\left(u_{1}\right) \cap A_{G}\left(u_{2}\right)\right|$, since when an elementary homomorphism $\varepsilon$ is applied to a graph $G, \varepsilon(G)$ will lose one edge for each vertex which is adjacent to both vertices that were identified, and all other edges will remain in $\varepsilon(G)$. Likewise, by the same reasoning as above, $\left|E\left(\theta_{\varepsilon}(\bar{G})\right)\right|=|E(\bar{G})|-\left|A_{\bar{G}}\left(u_{1}\right) \cap A_{\bar{G}}\left(u_{2}\right)\right|-1$, where the extra edge removed is the one which was contracted. Now if $(2.1)$ holds both $\varepsilon(G)$ and $\theta_{\varepsilon}(\bar{G})$ must contain the same number of edges. Thus

$$
|E(G)|-\left|A_{G}\left(u_{1}\right) \cap A_{G}\left(u_{2}\right)\right|=|E(\bar{G})|-\left|A_{\bar{G}}\left(u_{1}\right) \cap A_{\bar{G}}\left(u_{2}\right)\right|-1,
$$

where $|E(\bar{G})|=\frac{n(n-1)}{2}-|E(G)|$, which proves the lemma.

3. SELF-COMPLEMENTARY GRAPHS.

We first summarize some results from [4], [5] and [6] that will be needed. Let $S$ be a self-complementary graph. If $S$ is of order $n$ then $n \equiv 0,1(\bmod 4)$. Suppose $\mathrm{f}$ is an isomorphism such that $\mathrm{f}(\mathrm{S})=\overline{\mathrm{S}}$. If $\mathrm{n} \equiv 0(\bmod 4)$ then $\mathrm{f}$ has no fixed vertex, that is $\mathrm{f}(\mathrm{u}) \neq \mathrm{u}$ for $\mathrm{all} \mathrm{u} \in \mathrm{V}(\mathrm{S})$. However if $\mathrm{n} \equiv 1(\bmod 4)$ there exists precisely one fixed vertex for $\mathrm{f}$, and moreover any such $\mathrm{S}$ can be constructed by appropriately adding this fixed vertex, of valency $\frac{n-1}{2}$, to a self-complementary graph of order $\mathrm{n}-1$. Lastly there are regular self-complementary graphs of degree $\frac{\mathrm{n}-1}{2}$ if and only if $\mathrm{n} \equiv 1(\bmod 4)$.

We will assume throughout this section that $\mathrm{S}$ in nontrivial, that is $\mathrm{n}>1$. The following sequence of four lemmas will complete the proof of Theorem 1 .

LEMMA 2. If $\mathrm{S}$ is of order $\mathrm{n} \equiv 0(\bmod 4)$ then there exist vertices $u_{1}, u_{2} \varepsilon V(S)$ such that $\mathrm{u}_{1} \mathrm{u}_{2} \notin \mathrm{E}(\mathrm{S})$ and $\rho_{\mathrm{s}}\left(\mathrm{u}_{1}\right)=\rho_{\mathrm{s}}\left(\mathrm{u}_{2}\right)$.

PROOF. Since $\mathrm{n} \equiv 0(\bmod 4)$, any given valency that occurs in $\mathrm{S}$ will occur an even number of times, [4]. Thus there are vertices $u_{1}, u_{2} \varepsilon V(S)$ such that $\rho_{s}\left(u_{1}\right)=\rho_{s}\left(u_{2}\right)$. Now let $f(s)=\bar{s}$, then $\rho_{s}\left(u_{1}\right)=\rho_{s}\left(u_{2}\right)$ implies $\rho_{s}\left(f\left(u_{1}\right)\right)=\rho_{s}\left(f\left(u_{2}\right)\right)$ and also $\rho_{S}\left(f\left(u_{1}\right)\right)=\rho_{s}\left(f\left(u_{2}\right)\right)$. But $u_{1} u_{2} \varepsilon E(S)$ if and only if $f\left(u_{1}\right) f\left(u_{2}\right) \notin E(S)$ and so $u_{1} u_{2}$ or $f\left(u_{1}\right) f\left(u_{2}\right)$ satisy the lemma.

REMARK. For any self-complementary graph $S$ of order $n,|E(S)|=\frac{n(n-1)}{4}$. Thus from Lemma 1 , if $\varepsilon$ is an elementary homomorphism of $S$ which identifies the vertices $u_{1}$ and $u_{2}$ and $\varepsilon(S)=\theta_{\varepsilon}(\bar{S})$, then $\left|A_{s}\left(u_{1}\right) \cap A_{s}\left(u_{2}\right)\right|=\left|A_{s}^{-}\left(u_{1}\right) \cap A_{s}\left(u_{2}\right)\right|+1$.

LEMMA 3. If $\mathrm{S}$ is of order $\mathrm{n} \equiv 0(\bmod 4)$ then there is an elementary homomorphism $\varepsilon$ of $S$ such that $\varepsilon(S) \neq \theta_{\varepsilon}(\bar{S})$.

PROOF. Using Lemma 2 choose $u_{1}, u_{2} \varepsilon V(S)$ such that $u_{1} u_{2} \notin E(S)$ but $\rho_{s}\left(u_{1}\right)=\rho_{s}\left(u_{2}\right)$. Now for any $u \in V(S)$ that is distinct from $u_{1}$ and $u_{2}$, $u$ can be adjacent to both $u_{1}$ and $u_{2}$, neither $u_{1}$ nor $u_{2}$, or adjacent to one and not the other. 
Thus we have

$$
\begin{aligned}
& A_{s}\left(u_{1}\right)=\left\{u_{2}, \ldots, a_{k_{1}}, b_{1}, \ldots, b_{\ell}\right\} \quad A_{s}\left(u_{2}\right)=\left\{a_{1}, \ldots, a_{k_{1}}, c_{1}, \ldots, c_{\ell}\right\} \\
& A-\left(u_{1}\right)=\left\{u_{2}, d_{1}, \ldots, d_{k_{2}}, c_{1}, \ldots, c_{\ell}\right\} \quad A-\left(u_{2}\right)=\left\{u_{1}, d_{1}, \ldots, d_{k_{2}}, b_{1}, \ldots, b_{\ell}\right\}
\end{aligned}
$$

where $k_{1}=\left|A_{s}\left(u_{1}\right) \cap A_{s}\left(u_{2}\right)\right|$ and $k_{2}=\left|A_{s}\left(u_{1}\right) \cap A_{s}-\left(u_{2}\right)\right|$. Let $\varepsilon$ identify $u_{1}$ and $u_{2}$ and suppose $\varepsilon(S)=\theta_{\varepsilon}(\bar{S})$, so that from the proceding Remark $k_{1}=k_{2}+1$. Then

$$
n-1=\rho_{s}\left(u_{1}\right)+\rho-\left(u_{1}\right)=\left(k_{1}+\ell\right)+\left(k_{2}+1+\ell\right)=2 k_{1}+2 \ell .
$$

Hence $\mathrm{n}=2 \mathrm{k}_{1}+2 \ell+1 \neq 0(\bmod 4)$, a contradiction.

We now consider those self-complementary graphs of order $n \equiv 1$ (mod 4) and let $v$ be the fixed vertex, of valency $\frac{n-1}{2}$, under the isomorphism $f(S)=\bar{s}$.

LEMMA 4. Let $\mathrm{S}$ be of order $\mathrm{n} \equiv 1(\bmod 4)$ and $\varepsilon$ be an elementary homomorphism that identifies any $u \in V(S)$ with $v$. If $\varepsilon(S)=\theta_{\varepsilon}(\bar{S})$ then $S$ is regular.

PROOF. Let $f(S)=\bar{S}$, then for any $u^{\prime} \varepsilon V(S)$ distinct from $v, v u^{\prime} \varepsilon E(S)$ if and only if $\mathrm{v} f\left(\mathrm{u}^{\prime}\right) \notin \mathrm{E}(\mathrm{S})$ while $\rho_{\mathrm{S}}\left(\mathrm{u}^{\prime}\right)=\rho_{\mathrm{S}}\left(\mathrm{f}\left(\mathrm{u}^{\prime}\right)\right)$.

Suppose $S$ is not regular, then there is a vertex $u \in V(S)$ such that $\rho_{S}(u) \neq \frac{n-1}{2}$ and, from the observation above, $\mathrm{v} u \notin \mathrm{E}(\mathrm{S})$. Now let $\varepsilon$ identify this vertex $\mathrm{u}$ and $v$ and suppose $\varepsilon(S)=\theta_{\varepsilon}(\bar{S})$. We have, as in the proof of Lemma 3 ,

$$
\begin{array}{ll}
A_{s}(v)=\left\{a_{1}, \ldots, a_{k_{1}}, b_{1}, \ldots, b_{\frac{n-1}{2}}-k_{1}\right. & A_{s}(u)=\left\{a_{1}, \ldots, a_{k_{1}}, c_{1}, \ldots, c_{\ell}\right\} \\
A_{s}(v)=\left\{u, d_{1}, \ldots, d_{k_{2}}, c_{1}, \ldots, c_{\ell}\right\} & A_{s}(u)=\left\{v, d_{1}, \ldots, d_{k_{2}}, b_{1}, \ldots, b_{n-1}-k_{1}\right\}
\end{array}
$$

where $k_{1}=\left|A_{s}(u) \cap A_{s}(v)\right|$ and $k_{2}=\left|A_{s}(u) \cap A_{s}(v)\right|$. But $\rho-(v)=\frac{n-1}{2}$ and so $\ell=\frac{\mathrm{n}-1}{2}-\left(\mathrm{k}_{2}+1\right)$, and $\mathrm{k}_{1}=\mathrm{k}_{2}+1$ since $\varepsilon(\mathrm{S})=\theta_{\varepsilon}(\overline{\mathrm{S}})$. Hence

$\rho_{S}(u)=k_{1}+\ell=k_{1}+\frac{n-1}{2}-\left(k_{2}+1\right)=\frac{n-1}{2}$, a contradiction.

LEMMA 5. If $S$ is a regular self-complementary graph then there is an elementary homomorphism $\varepsilon$ such that $\varepsilon(S) \neq \theta_{\varepsilon}(\bar{S})$

PROOF. First note that $S \mathrm{v}$ is self-complementary, with $\frac{\mathrm{n}-1}{2}$ vertices of valency $\frac{n-1}{2}$ and $\frac{n-1}{2}$ vertices of valency $\frac{n-3}{2}$. Thus there are $\frac{n-1}{2}$ edges joining every vertex of valency $\frac{n-1}{2}$ to the vertices of valency $\frac{n-3}{2}$, and these must appear in either $S \mathrm{v}$ of $\overline{\mathrm{S} v}$. Since $\mathrm{S} v=\overline{\mathrm{S} v}$ these edges must be equally divided between $S \mathrm{v}$ and its complement. When $\mathrm{v}$ is added to $\mathrm{S} v$ to form $S$ it is adjacent to every vertex of valency $\frac{n-3}{2}$. Thus if $u \in V(S v)$ such that $\rho_{S}(u)=\frac{n-1}{2}$, we have $\left|A_{s}(u) \cap A_{s}(v)\right|=\frac{n-1}{4}$.

Now $S$ is regular of degree $\frac{n-1}{2}$ and we let $\varepsilon$ identify $u$, as described above, and $\mathrm{v}$. Assume $\varepsilon(\mathrm{S})=\theta_{\varepsilon}(\bar{S})$ and $\mathrm{u}^{\prime} \varepsilon \mathrm{V}(\varepsilon(G))$ is the image of u and $\mathrm{v}$ under $\varepsilon$. Then

$$
\begin{aligned}
& \rho_{\varepsilon}(s)(u) \\
&=\rho_{s}(u)+\rho_{s}(v)-\left|A_{s}\left(u_{1}\right) \cap A_{s}\left(u_{2}\right)\right| \\
&=\frac{n-1}{2}+\frac{n-1}{2}-\frac{n-1}{4}=3\left(\frac{n-1}{4}\right),
\end{aligned}
$$


since every vertex adjacent to both $u$ and $v$ in $S$ will only account for one edge incident to $\mathrm{u}^{\prime}$ in $\varepsilon(\mathrm{S})$. Similarly

$$
\rho_{\theta}(\bar{s})\left(u^{\prime}\right)=\rho_{\bar{s}}(u)+\rho_{\bar{s}}(v)-\left|A_{\bar{s}}(u) \cap A_{s}(v)\right|-2,
$$

where the 2 is substracted since the edge contracted under $\theta_{\varepsilon}$ is incident to both $u$ and $v$. However since $\varepsilon(S)=\theta_{\varepsilon}(\bar{S})$ we must have $\left|A_{s}(u) \cap A_{s}(v)\right|=\left|A_{S}(u) \cap A_{S}(v)\right|+1$, and so

$$
\rho_{\theta_{\varepsilon}(\overline{\mathrm{s}})}\left(\mathrm{u}^{\prime}\right)=\frac{\mathrm{n}-1}{2}+\frac{\mathrm{n}-1}{2}-\frac{\mathrm{n}-1}{4}-1=3\left(\frac{\mathrm{n}-1}{4}\right)-1 .
$$

Every other $\bar{u} \varepsilon V\left(\theta_{\varepsilon}(\bar{S})\right)$ has valency $\rho_{\bar{s}}(\bar{u})-1=\frac{n-3}{2}$ or $\rho_{\bar{s}}(\bar{u})=\frac{n-1}{2}$ depending on whether or not $\bar{u} \varepsilon A_{-}(u) \cap A_{-s}(v)$. Therefore $\rho_{\varepsilon(s)}\left(u^{\prime}\right)>\rho_{\theta_{\varepsilon}}(\bar{s})(\bar{u})$ for every vertex $\overline{\mathrm{u}}$ in $\theta_{\varepsilon}(\overline{\mathrm{S}})$, and hence $\varepsilon(\mathrm{S}) \neq \theta_{\varepsilon}(\overline{\mathrm{S}})$ since isomorphisms must preserve valencies.

Now that the proof of Theorem 1 is complete we will show that for every selfcomplementary graph there is a homomorphism $\phi$ which satisfies (1.1).

LEMMA 6. Let $\mathrm{S}$ be of order $\mathrm{n}$ and $\mathrm{f}(\mathrm{S})=\overline{\mathrm{S}}$. Then in the set $V=\left\{\left(u_{i} f\left(u_{i}\right)\right) \mid u_{i} \varepsilon V(S), i=1, \ldots, n\right\}$ precisely $m=\left[\frac{n}{2}\right]$ are non-adjacent pairs of distinct vertices in $S$.

PROOF. Let $u \in V(S)$ such that $f(u) \neq u$. Then $u$ and $f(u)$ are adjacent in $S$ if and only if $f(u)$ and $f(f(u))$ are non-adjacent in $S$. Now $f$ is an isomorphism, and so $f(u)$ and $f(f(u))$ must also be distinct vertices of $s$. Hence for every pair of distinct non-adjacent vertices in $\mathrm{V}$, there is a pair of adjacent vertices in $\mathrm{V}$ and conversely. Thus there must be $m=\left[\frac{n}{2}\right]$ pairs of distinct non-adjacent vertices in $v$. THEOREM 5. Let $\mathrm{S}$ be of order $\mathrm{n}$ and $\mathrm{m}=\left[\frac{\mathrm{n}}{2}\right]$. There exists a homomorphism $\phi$ of $S$ such that $\phi_{(}(S)=K_{n-m}=\theta_{\phi}(\bar{S})$.

PROOF. For each of the $m$ pairs of distinct non-adjacent vertices provided by Lemma 6 , there exists an elementary homomorphism $\varepsilon_{i}$ of $s$ that identifies $u_{i}$ and $f\left(u_{i}\right)$. Let $\phi=\varepsilon_{1}, \varepsilon_{2}, \ldots, \varepsilon_{\mathrm{m}}$ so that both $\phi(S)$ and $\theta_{\phi}(\bar{S})$ will have $n-m$ vertices. Suppose $\phi(S) \neq K_{n-m}$, then there are vertices $u_{1}, u_{2} \varepsilon V(\phi(S))$ such that $\mathrm{u}_{1} \mathrm{u}_{2} \notin \mathrm{E}(\phi(\mathrm{s}))$. Let $\phi^{-1}\left(\mathrm{u}_{1}\right)=\left\{\mathrm{a}_{1}, \mathrm{a}_{2}\right\}$ and $\phi^{-1}\left(\mathrm{u}_{2}\right)=\left\{\mathrm{b}_{1}, \mathrm{~b}_{2}\right\}$. Thus $\mathrm{a}_{1} \neq \mathrm{b}_{1}$, $\mathrm{f}\left(\mathrm{a}_{1}\right)=\mathrm{a}_{2}$ and $\mathrm{f}\left(\mathrm{b}_{1}\right)=\mathrm{b}_{2}$ where $\mathrm{f}(\mathrm{S})=\overline{\mathrm{s}}$. Now $\mathrm{u}_{1} \mathrm{u}_{2} \notin \mathrm{E}(\phi(\mathrm{S}))$ implies $\mathrm{a}_{1} \mathrm{~b}_{1} \notin \mathrm{E}(\mathrm{S})$ and so $f\left(a_{1}\right)\left(B_{1}\right) \notin E(\bar{S})$. But then $f\left(a_{1}\right) f\left(b_{1}\right)=a_{1} b_{2} \varepsilon E(S)$ and so $u_{1} u_{2} \varepsilon E(\phi(S))$, a contradiction. Thus $\phi(S)=\mathrm{K}_{\mathrm{n}-\mathrm{m}}$. By an argument virtually identical to the one just given it follows that $\theta_{\phi}(\bar{S})=K_{n-m}$, which proves the theorem.

Erom [3] we know that the smallest homomorphic image of any graph $G$ is a complete graph of order $\chi(G)$. Thus from Theorem 5 we have $\chi(S) \leqq n-\left[\frac{n}{2}\right]$ for every selfcomplementary graph $\mathrm{S}$ of order $\mathrm{n}$. However if a graph is contractable to a complete graph of order $t$, then it has a complete contraction of order $k$ for $1 \leqq k \leqq t$, also from [3]. Hence Theorem 5 shows that every self-complementary graph satisfies Hadwiger's Conjecture [2], that is, every self-complementary graph $S$ has a complete contraction of order $x(S)$.

4. CONCLUDING REMARKS.

It would certainly be desirable to find some simple necessary and sufficient 
conditions on the graph G, and perhaps on $\bar{G}$, to ensure the existence of a homomorphism $\phi$ such that the graph equation $\phi(G)=\theta_{\phi}(\bar{G})$ holds. Given that such conditions can be found, we would like to be able to construct the appropriate homomorphisms for which the equation holds or perhaps enumerate those homomorphisms of $G$ that satisfy the equation. All of these problems remain open.

\section{REFERENCES}

1. GIRSE, R. Homomorphisms of Complete n-Partite Graphs, Internat. J. Math. Math. Sci., to appear.

2. HADWIGER, H. Uber eine Klassifikation der Streckenkomplexe, Vierteljschr. Naturforsch. Ges. Zurich 88 (1943), 133-142.

3. HARARY, F. HEDETNIEMI, S. and PRINS, G. An Interpolation Theorem for Graphical Homomorphisms, Portugal Math. 26 (1969), 453-462.

4. MORRIS, P. Self-Complementary Graphs and Digraphs, Math. Comp. 27 (1973), $216-217$.

5. RINGEL, G. Selbstkomplementare Graphen, Archiv d. Math. 14 (1963), 354-358.

6. SACHS, H. Uber Selbstkomplementare Graphen, Publ. Math. Debrecen 9 (1962), 270-288. 


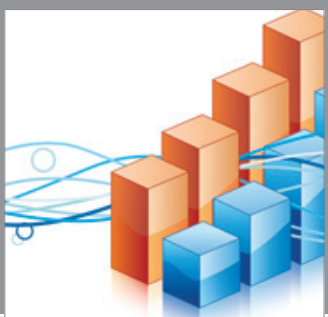

Advances in

Operations Research

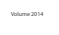

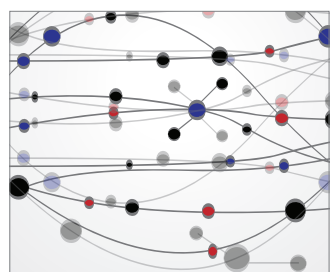

\section{The Scientific} World Journal
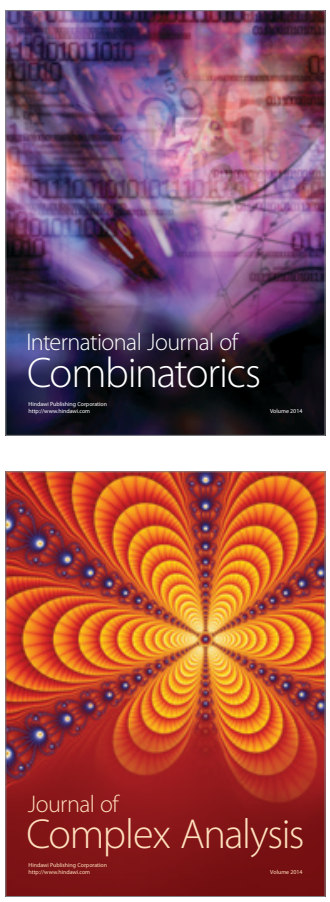

International Journal of

Mathematics and

Mathematical

Sciences
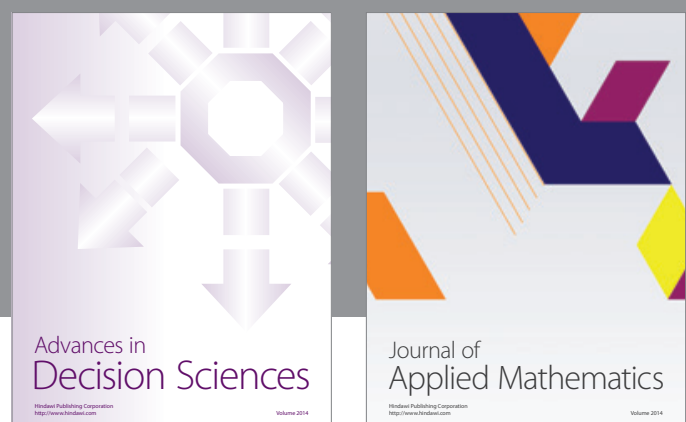

Journal of

Applied Mathematics
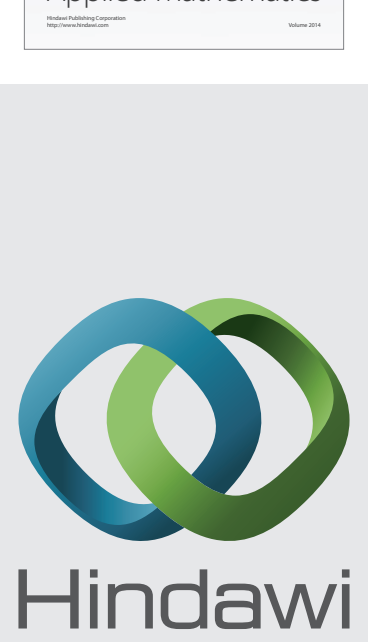

Submit your manuscripts at http://www.hindawi.com
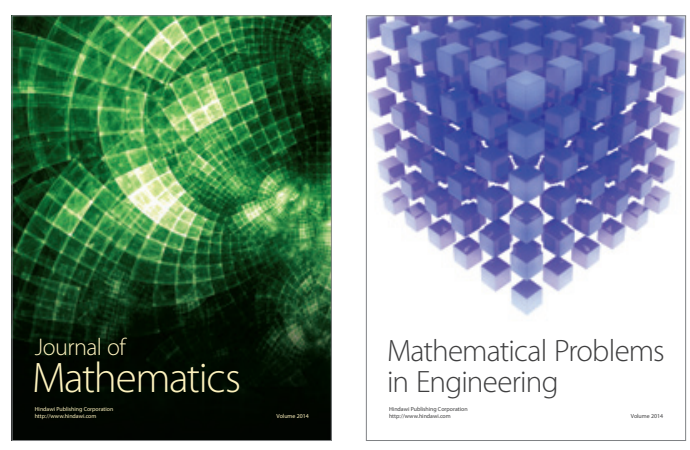

Mathematical Problems in Engineering
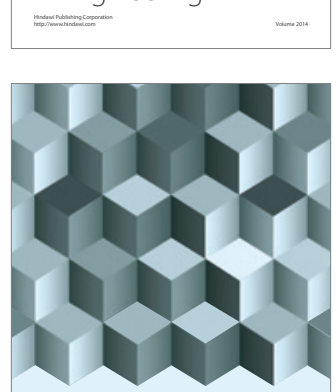

Journal of

Function Spaces
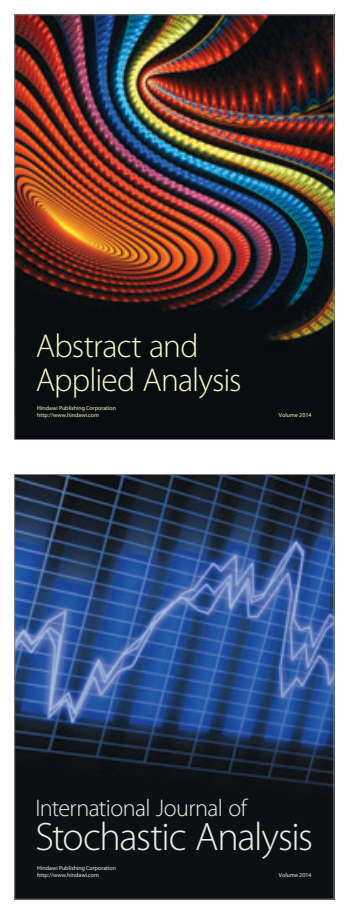

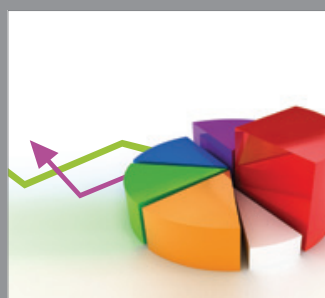

ournal of

Probability and Statistics

Promensencen
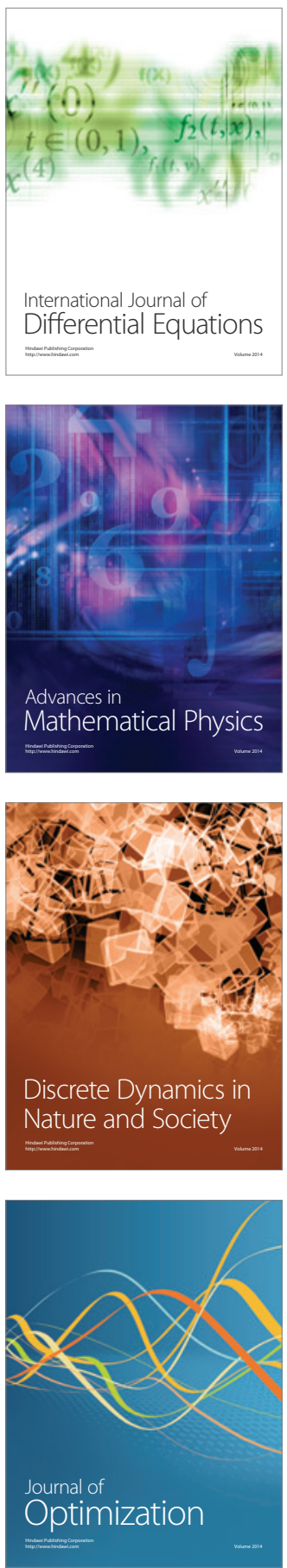